EPJ Web of Conferences 59, 01005 (2013)

DOI: $10.1051 /$ epjconf/20135901005

(C) Owned by the authors, published by EDP Sciences, 2013

\title{
Studies on shock ignition targets for inertial fusion energy
}

\author{
S. Atzeni ${ }^{1}$, a, A. Schiavi ${ }^{1}$, A. Marocchino ${ }^{1}$, A. Giannini ${ }^{1}$, A. Mancini ${ }^{1}$ \\ and M. Temporal ${ }^{2}$ \\ ${ }^{1}$ Dipartimento SBAI, Università di Roma La Sapienza and CNISM, Roma, Italy \\ ${ }^{2}$ ETSIA, UPM, Madrid, Spain
}

\begin{abstract}
Shock-ignited inertial fusion targets are studied by one dimensional and two-dimensional numerical simulations. Most of the study refers to the simple all-DT HiPER baseline target (imploded mass of $0.29 \mathrm{mg}$ ); both the reference laser wavelength $\lambda=0.35 \mu \mathrm{m}$, and $\lambda=0.25 \mu \mathrm{m}$ are considered. The target achieves 1D gain about $80(120)$ with total laser energy of $260 \mathrm{~kJ}(180 \mathrm{~kJ})$ at $\lambda=0.35 \mu \mathrm{m}(0.25 \mu \mathrm{m})$. Operating windows for the parameters of the laser ignition spike are described. According to preliminary simulations, gain 80-100 is also obtained by a scaled target (imploded mass of $1.8 \mathrm{mg}$ ) driven by $1.5 \mathrm{MJ}$ of green laser light $(0.53 \mu \mathrm{m})$. Two dimensional simulations indicate robustness to irradiation nonuniformities, and high sensitivity to target mispositioning. This can however be reduced by increasing the power of the ignition spike.
\end{abstract}

\section{INTRODUCTION}

Shock ignition $[1,2]$ is a recently proposed inertial confinement fusion (ICF) scheme, in which distinct pulses are used to precompress the fuel and to generate the central ignition hot spot. Implosion of the target is caused by a conventional time-shaped laser pulse, with peak intensity $I \leq 5 \times 10^{14} \mathrm{~W} / \mathrm{cm}^{2}$. Towards the end of the implosion a more intense $\left(I \simeq 5 \times 10^{15} \mathrm{~W} / \mathrm{cm}^{2}\right)$, shorter pulse produces an ablation pressure about $300 \mathrm{Mbar}$ and drives a strong converging spherical shock wave (laser wavelength $\lambda=0.35 \mu \mathrm{m}$ is assumed here). This shock wave eventually leads to multiplication of the central pressure by a factor 3-4, and to hot spot ignition. The advantages of this ICF scheme with respect to the conventional one are related to the reduction of the implosion velocity $u_{i}$, which is discussed in Sec. 2. Lowering $u_{\mathrm{i}}$ relaxes instability issues, and can also lead to substantially higher gain.

Shock-ignited targets have been studied by several authors [1-8]. In a previous paper [9] we have analyzed a simple all-DT target, driven by pulses with $\lambda=0.35 \mu \mathrm{m}$ and total energy of $250-300 \mathrm{~kJ}$. Here, we extend such a study. 1D and 2D results, obtained with the DUED code [10], are presented in Secs. 3 and 4, respectively. Performance of the HiPER all-DT target $[11,12]$ is studied for $\lambda=0.35 \mu \mathrm{m}$ as well as $\lambda=0.25 \mu \mathrm{m}$. Results for a CH-DT target proposed by the CELIA group [13] are also presented. We also show that a bigger target, upscaled from the HiPER target, achieves 1D gain of $80-100$ when irradiated by $1.5 \mathrm{MJ}$ pulses of green laser light $(\lambda=0.53 \mu \mathrm{m})$. In Sec. 4 we study the effects of nonuniform irradiation and target mispositioning. Conclusions are drawn in Sec. 5.

\section{IMPLOSION VELOCITY FOR IGNITION}

The configuration of the stagnating fuel of a shock-ignited target is shown in Fig. 1. The relevant ignition condition (see Fig. 1 and Ref. [14]) can then be written as a condition on the hot spot

\footnotetext{
${ }^{a}$ e-mail: stefano.atzeni@uniroma1.it
}

This is an Open Access article distributed under the terms of the Creative Commons Attribution License 2.0, which permits unrestricted use, distribution, and reproduction in any medium, provided the original work is properly cited. 
EPJ Web of Conferences
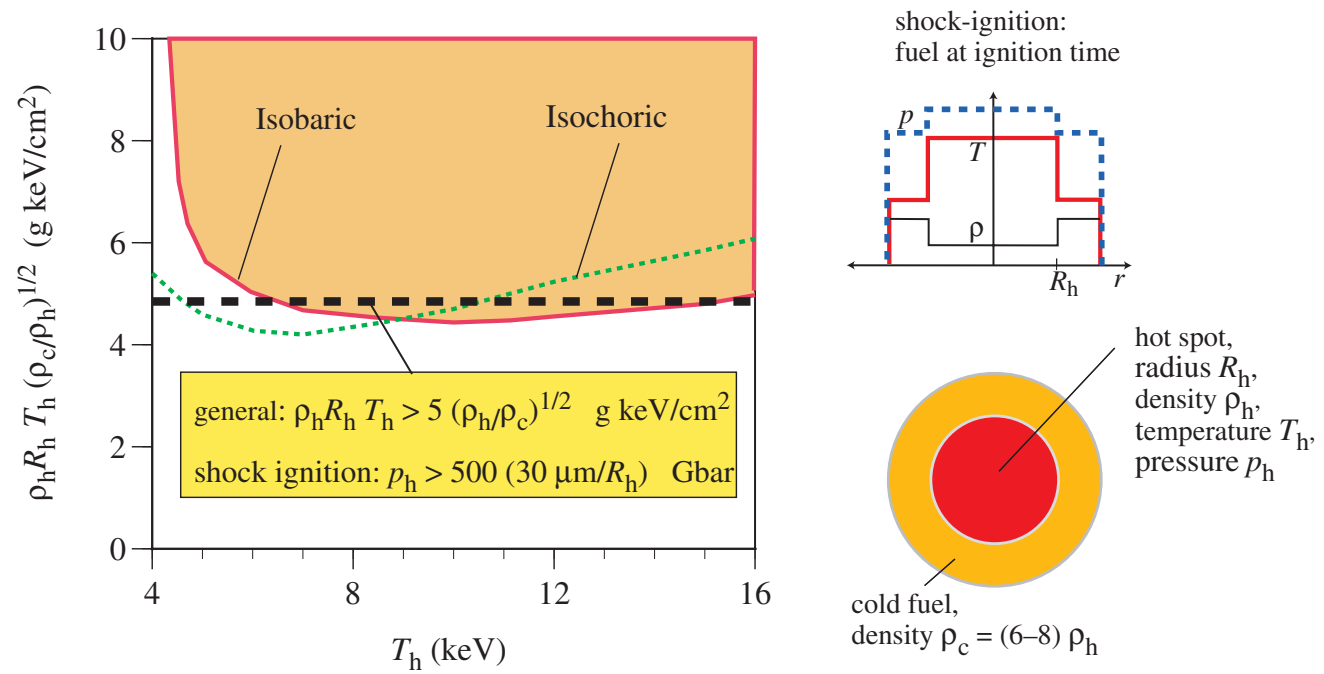

cold fuel,

density $\rho_{\mathrm{c}}=(6-8) \rho_{\mathrm{h}}$

Figure 1. Hot spot ignition region (orange region) and hot spot ignition condition: quantity $\rho_{\mathrm{h}} R_{\mathrm{h}} T_{\mathrm{h}}\left(\rho_{\mathrm{c}} / \rho_{\mathrm{h}}\right)^{1 / 2}$ vs hot spot temperature $T_{\mathrm{h}}$. The ignition condition for shock ignition refers to the fuel configuration sketched on the right-hand-side of the figure.

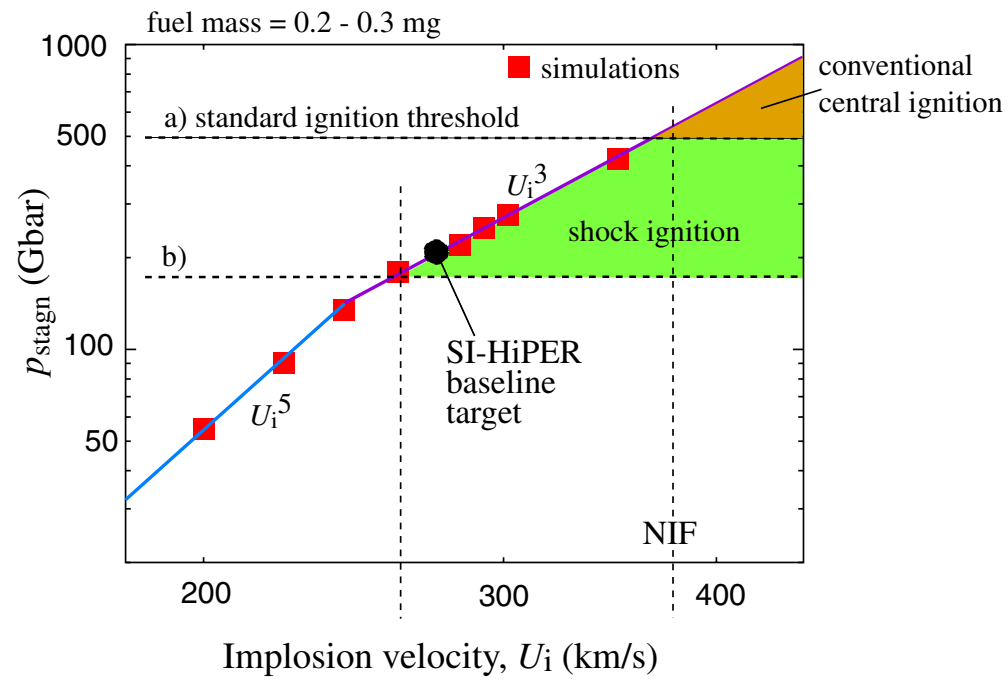

Figure 2. Stagnation pressure (no alpha-particle heating) vs implosion velocity. Simulation data (squares) refer to the HiPER baseline target. For this target standard central ignition requires stagnation pressure of about $500 \mathrm{Gbar}$ (line a), i.e. implosion velocity larger $370 \mathrm{~km} / \mathrm{s}$. In shock ignition the final stagnation pressure is multiplied by a factor of three by shock collisions, the equivalent no-shock stagnation pressure is somewhat below 200 Gbar (line b) and the velocity threshold for ignition is about $260 \mathrm{~km} / \mathrm{s}$.

pressure $p_{\mathrm{h}}$ :

$$
p_{\mathrm{h}}>\frac{500 \mathrm{Gbar}}{R_{\mathrm{h}} /(30 \mu \mathrm{m})} .
$$



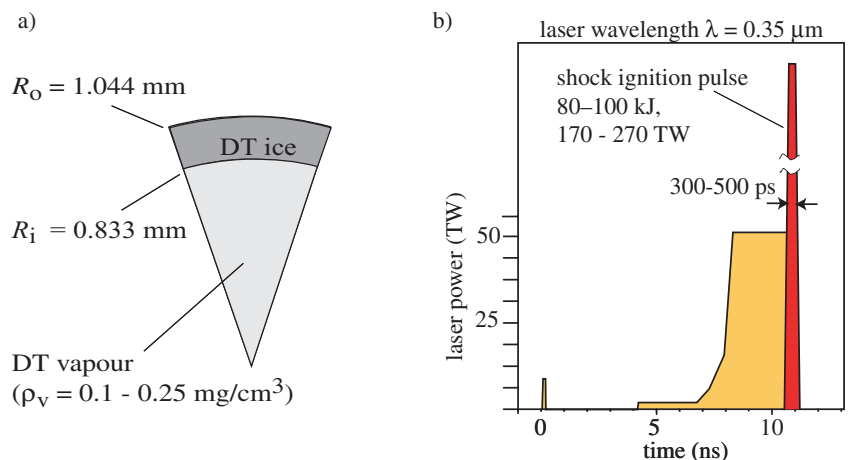

Figure 3. a) HiPER baseline target; b) laser pulse temporal profile (for laser wavelength $\lambda=0.35 \mu \mathrm{m}$ ).

On the other hand, the fuel pressure at stagnation depends strongly on the implosion velocity (see Fig. 2; see also Refs. [15, 16] and [8]). Pressure enhancement by shock collisions therefore lowers the requested implosion velocity. For targets with fuel mass of $0.2-0.3 \mathrm{mg}$ and isentrope parameter $\alpha=1.2-1.4$ the velocity threshold is reduced from $370 \mathrm{~km} / \mathrm{s}$ to $260 \mathrm{~km} / \mathrm{s}$.

Table 1. HiPER baseline target. Design constraints, pulse parameters and 1D target performance.

\begin{tabular}{|c|c|c|c|}
\hline & \multicolumn{2}{|c|}{ HiPER baseline target } & CELIA target \\
\hline \\
\hline \multicolumn{4}{|l|}{$\begin{array}{l}\text { Design constralnts } \\
\text { IFAR }\end{array}$} \\
\hline$<$ fuel adiabat index $>$ & \multicolumn{2}{|c|}{$\begin{array}{r}\leq 30 \\
\simeq 1.2\end{array}$} & \\
\hline RTI growth factor & \multicolumn{2}{|c|}{$\leq \exp (6)$} & \\
\hline$I \lambda^{2}$ (compr. pulse) & \multicolumn{2}{|c|}{$\leq 5 \times 10^{13} \mathrm{~W} / \mathrm{cm}^{2}(\mu \mathrm{m})^{2}$} & \\
\hline Laser wavelength $\lambda$ & $0.35 \mu \mathrm{m}$ & $0.25 \mu \mathrm{m}$ & $0.35 \mu \mathrm{m}$ \\
\hline \multicolumn{4}{|l|}{ compression pulse } \\
\hline spot width $w_{\mathrm{c}}$ & $640 \mu \mathrm{m}$ & $640 \mu \mathrm{m}$ & $680 \mu \mathrm{m}$ \\
\hline flat-top power $P_{\mathrm{c}}$ & $42-50 \mathrm{TW}$ & $36 \mathrm{TW}$ & $80 \mathrm{TW}$ \\
\hline energy $E_{\mathrm{c}}$ & $164-180 \mathrm{~kJ}$ & $140 \mathrm{~kJ}$ & $250 \mathrm{~kJ}$ \\
\hline absorption efficiency $\eta_{\mathrm{a}}-\mathrm{c}$ & $74 \%$ & & $90 \%$ \\
\hline \multicolumn{4}{|l|}{ Ignition spike } \\
\hline spot width $w_{\mathrm{s}}$ & $400 \mu \mathrm{m}$ & $400 \mu \mathrm{m}$ & $345 \mu \mathrm{m}$ \\
\hline power $P_{\mathrm{s}}$ & $>150 \mathrm{TW}$ & $>70 \mathrm{TW}$ & $>140 \mathrm{TW}$ \\
\hline energy $E_{\mathrm{s}}$ & $>80 \mathrm{~kJ}$ & $>40 \mathrm{~kJ}$ & $>60 \mathrm{~kJ}$ \\
\hline absorption efficiency $\eta_{\mathrm{a}-\mathrm{s}}$ & $43 \%$ & & $70 \%$ \\
\hline synchronization & $120 \mathrm{ps}\left(P_{\mathrm{s}}=170 \mathrm{TW}\right)$ & $250 \mathrm{ps}\left(P_{\mathrm{s}}=75 \mathrm{TW}\right)$ & $250 \mathrm{ps}\left(P_{\mathrm{s}}=200 \mathrm{TW}\right)$ \\
\hline & $250 \mathrm{ps}\left(P_{\mathrm{s}}=270 \mathrm{TW}\right)$ & & \\
\hline \multicolumn{4}{|l|}{ Compression results (no spike) } \\
\hline Implosion velocity & $285 \mathrm{~km} / \mathrm{s}$ & & $245 \mathrm{~km} / \mathrm{s}$ \\
\hline$<\rho R>$ & $1.5 \mathrm{~g} / \mathrm{cm}^{2}$ & & $1.9 \mathrm{~g} / \mathrm{cm}^{2}$ \\
\hline Imploded fuel mass & $0.29 \mathrm{mg}$ & & $0.36 \mathrm{mg}$ \\
\hline \multicolumn{4}{|l|}{ Fusion performance } \\
\hline Fusion energy & $\leq 24 \mathrm{MJ}$ & $\leq 22 \mathrm{MJ}$ & $\leq 33 \mathrm{MJ}$ \\
\hline 1D Gain & $\leq 80$ & $\leq 120$ & $\leq 100$ \\
\hline Hot spot convergence & $35-42$ & & $30-42$ \\
\hline
\end{tabular}




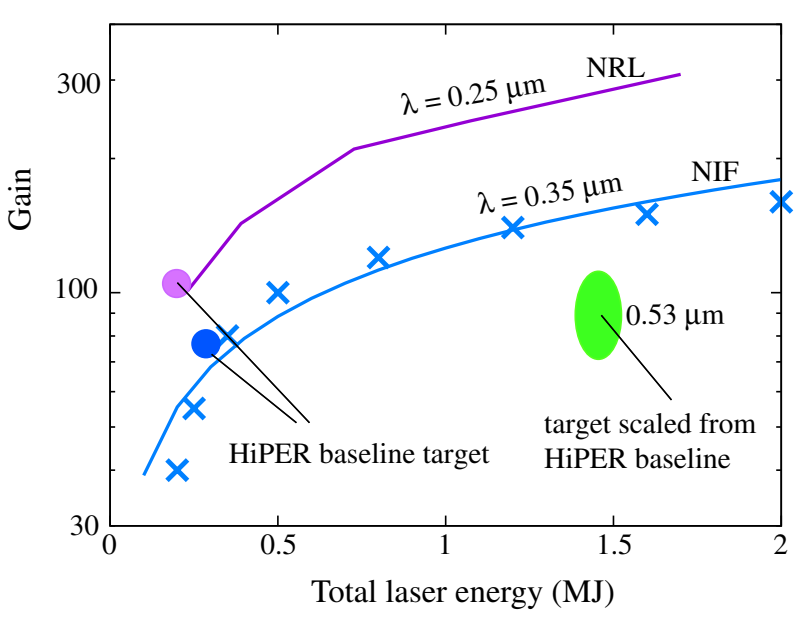

Figure 4. Gain computed for the HiPER baseline target driven by laser light with wavelength $\lambda=0.25 \mu \mathrm{m}$ and $\lambda=0.35 \mu \mathrm{m}$ (filled circles), and for a scaled target, driven by light with wavelength $\lambda=0.53 \mu \mathrm{m}$ (green area). These results are compared with recently published data by Schmitt et al. (curve labelled NRL, [7]), Perkins et al. (curve labelled NIF, [5]), Lafon et al. (crosses, [8]).

\section{TARGET DESIGN AND 1D PERFORMANCE}

Most of our studies refer to the HiPER baseline target [11], shown in Fig. 3; see also Ref. [9]. Design constraints, main pulse parameters and performance data based on 1D simulations are listed in Table 1. Laser wavelengths of $0.35 \mu \mathrm{m}$ and $0.25 \mu \mathrm{m}$ are considered. The table shows that gains up to 80 (120) are computed for drive energy below $300 \mathrm{~kJ}(200 \mathrm{~kJ})$ at $\lambda=0.35 \mu \mathrm{m}(0.25 \mu \mathrm{m})$. High resolution $2 \mathrm{D}$ single mode simulations confirm that the initial laser picket is required to reduce growth of Rayleigh-Taylor instability (RTI) at the ablation front [17]. The table also shows our results for a target with DT fuel and $\mathrm{CH}$ ablator proposed by the CELIA group [13] (see also Ref. [18]). This target has inner radius of $670 \mu \mathrm{m}$, a $200 \mu \mathrm{m}$ thick DT layer, and a $28 \mu \mathrm{m}$ outer CH layer.

A large parametric scan (thousands of runs) indicates that the HiPER target tolerates errors of $\pm 3-5 \%$ in energy, power, mass, and of $\pm 1 \%$ in target radius.

The baseline target cannot be ignited with green laser light $(\lambda=0.53 \mu \mathrm{m})$, if the same constraints as in Table 1 are imposed on compression laser intensity $\left(I \lambda^{2} \leq 5 \times 10^{13} \mathrm{~W} / \mathrm{cm}^{2} \mu \mathrm{m}^{2}\right)$ and on RTI growth. Indeed achievable implosion velocity and then stagnation pressure decrease with increasing $\lambda$. However, according to Eq. (1) the pressure required for ignition is $p_{\mathrm{h}} \propto R_{\mathrm{h}}^{-1} \propto m^{-1 / 3}$, assuming geometrically scaled imploded assemblies. (Here $m$ is the fuel mass.) This suggests the design of bigger targets. We have indeed found that a target with total fuel mass of about $3.1 \mathrm{mg}$ (and imploding payload of $1.8 \mathrm{mg}$ ) can be shock-ignited by a green light pulse of about $1.5 \mathrm{MJ}$, with peak compression laser power of about $90 \mathrm{TW}$ and spike power of about $550 \mathrm{TW}$. According to preliminary 1D simulations, gain up to 100 can be achieved. The parameter $I \lambda^{2}$ is just about 10-20\% larger than for the baseline target (both at compression and at ignition spike). Details of this on-going work will be presented elsewhere.

Gain computed for the above targets are compared in Fig. 4 with gain curves and gain points obtained by other authors $[5,7,8]$.

\section{IRRADIATION NONUNIFORMITIES AND TARGET MISPOSITIONING}

The accurate study of the effect of irradiation nonuniformities requires full 3D simulations. However, for a first, necessarily approximated study, we have performed 2D simulations using a simplified treatment of laser interaction and irradiation pattern [9]. We assume radial rays (with power adjusted 


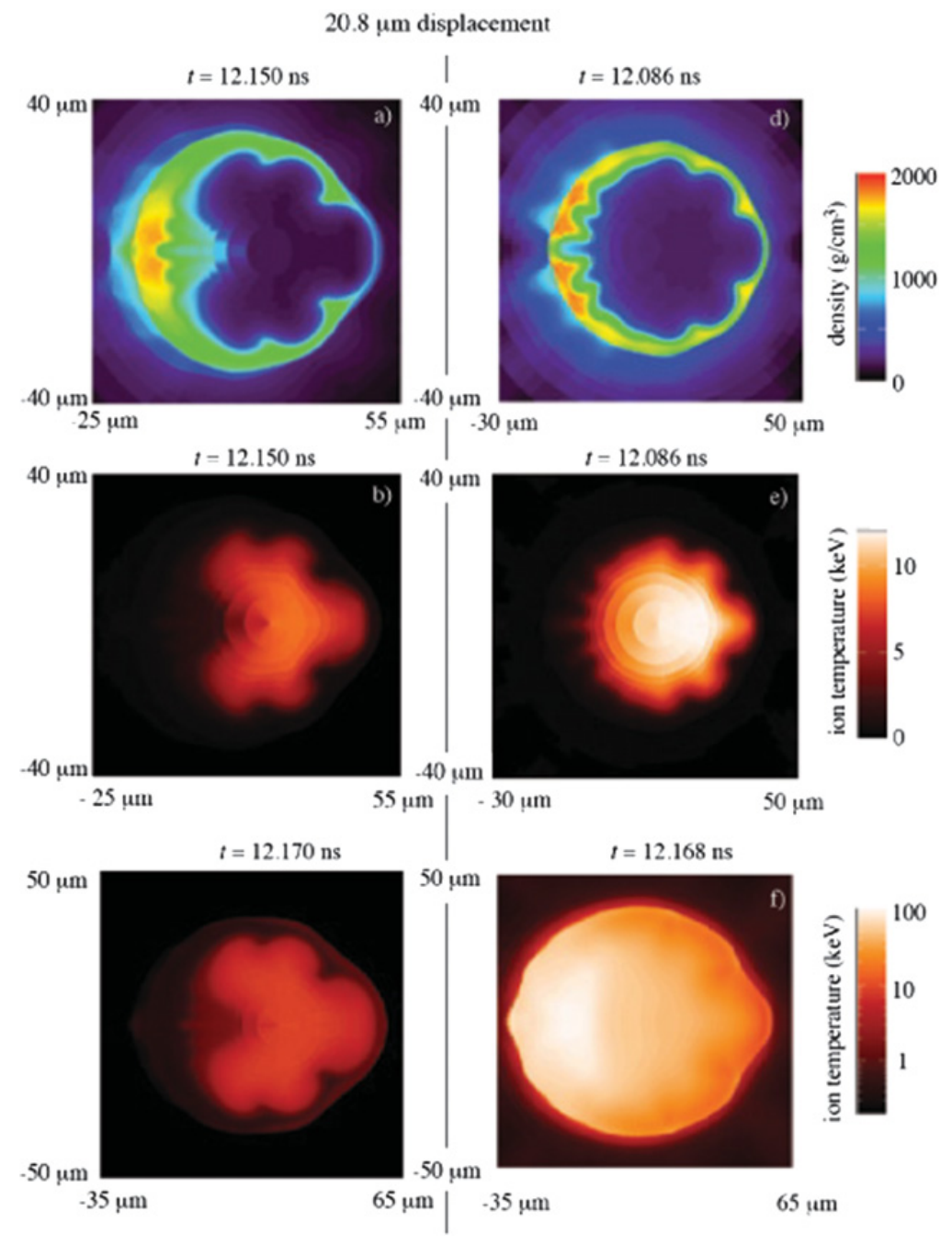

Figure 5. Simulation of HiPER baseline targets displaced by $20.8 \mu \mathrm{m}$ along the polar axis (horizontal in the figure), compressed by a 48 (perfect) beam laser. Left column: nominal ignition spike. The target does not ignite. Right column: ignition spike with power increased by $50 \%$. The target ignites releasing about the $90 \%$ of the nominal yield. (a) and (d): density maps at stagnation; (b) and (e): ion temperature maps at the same times as in (a) and (d), respectively; (c) and (f): ion temperature maps at the respective times of peak temperature. Coordinate system centered at the target center at $t=0$.

to produce the same implosion as computed by $1 \mathrm{D}$ runs with $2 \mathrm{D}$ raytracing) and power distribution with a time independent angular nonuniformity spectrum corresponding to the initial illumination spectrum computed for the 48-beam HiPER reference irradiation scheme (see Refs. [19] and [12]). Previous simulations [9] have shown that hot spot deformations are tolerable at ignition for the nominal scheme. Instead, our previous study showed that on-axis target mispositioning must be limited to about $15 \mu \mathrm{m}$ (i.e. $1.5 \%$ of the radius). Subsequent simulations have however shown that sensitivity to mispositioning can be relaxed by increasing the spike power. An example is shown in Fig. 5.

We have also found that large asymmetries of the spike power are tolerated (See Ref. [18]). Indeed simulations show that thermal conductivity between critical density and ablation front smooths out pressure nonuniformity. However, this result must be confirmed by simulations with models of electron 
transport more accurate than the presently used flux-limited Spitzer conductivity. To this purpose, we are testing nonlocal electron transport treatments, and plan to include one of them in DUED.

The reference irradiation scheme minimizes the initial illumination nonuniformity. On the other hand, we have shown that this is quite unstable, since it is highly sensitive to laser errors (imbalance, mispointing) and target positioning [20]. More stable schemes have been proposed on the basis of simple illumination studies. Such schemes employ beams with larger focal spots and high-order supergaussian intensity profiles. Their accurate study however requires fully 3-D treatment of both laser beams and laser ray-tracing, which have recently been included in DUED and are currently being validated.

\section{CONCLUSIONS}

We have studied simple shock ignition targets by means of $1 \mathrm{D}$ and $2 \mathrm{D}$ simulations. According to $1 \mathrm{D}$ simulations they can achieve high gain at laser energy of a few hundred kJ, and laser wavelengths $\lambda$ of 0.35 or $0.25 \mu \mathrm{m}$. 1D gain about 100 is also computed for a target driven by $1.5 \mathrm{MJ}$ of green light $(\lambda=0.53 \mu \mathrm{m})$. Several aspects of target robustness have been analyzed. The present results support the potentials of shock ignition for fusion energy production. However, one should be aware of the preliminary nature of current studies. Assessment of shock ignition requires, first of all, the demonstration of the efficient generation of 300 Mbar shocks. Experiments can be performed at existing large laser facilities. Additional specific issues concern laser-plasma instabilities driven by the intense ignition spike [21], and very low entropy stable acceleration. Concerning hydrodynamic simulations, like those presented in this paper, the next steps are the study of more realistic targets and irradiation schemes (eg the polar direct drive schemes feasible at NIF [22]).

We thank G. Schurtz and X. Ribeyre for many discussions and for providing us data on the proposed CELIA target. Work partially supported by the Italian MIUR project PRIN 20072KW45J and by HiPER project and Preparatory Phase Funding Agencies (EC, MSMT and STFC). Part of the simulations where performed thanks to a CASPUR grant.

\section{References}

[1] R. Betti et al., Phys. Rev. Lett. 98, 155001 (2007)

[2] R. Betti et al., J. Phys. Conf. Ser. 112, 022024 (2008)

[3] X. Ribeyre et al., Plasma Phys. Control. Fusion 51, 015013 (2009)

[4] X. Ribeyre et al., Plasma Phys. Control. Fusion 51, 124030 (2009)

[5] L.J. Perkins, R. Betti, K.N. LaFortune, W.H. Williams, Phys. Rev. Lett. 103, 045004 (2009)

[6] B. Canaud, M. Temporal, New J. Phys. 12, 043037 (2010)

[7] A.J. Schmitt, J. W. Bates, S. P. Obenschain, S.T. Zalesak, D.E. Fyfe, Phys. Plasmas 17, 042701 (2010)

[8] M. Lafon, X. Ribeyre, G. Schurtz, Phys. Plasmas 17, 052704 (2010)

[9] S. Atzeni, A. Schiavi, A. Marocchino, Plasma Phys. Control. Fusion 35, 035010 (2011)

[10] S. Atzeni et al., Computer Phys. Commun. 169, 153 (2005)

[11] S. Atzeni, A. Schiavi, C. Bellei, Phys. Plasmas 14, 052702 (2007)

[12] S. Atzeni et al., Nucl. Fusion 49, 055008 (2009)

[13] G. Schurtz, X. Ribeyre, CELIA Laboratory, Bordeaux, private communication (May 2010)

[14] S. Atzeni, J. Meyer-ter-Vehn, The Physics of Inertial Fusion (Clarendon, Oxford 2004)

[15] J. Meyer-ter-Vehn, C.Z. Schalk, Z. Nat. forsch. A37, 955 (1982)

[16] A. Kemp, J. Meyer-ter-Vehn, S. Atzeni, Phys. Rev. Lett. 86, 3336 (2001) 


\section{IFSA 2011}

[17] A. Marocchino, S. Atzeni, A. Schiavi, Phys. Plasmas 17, 112703 (2010)

[18] S. Atzeni, G. Schurtz, HiPER WP9, Proc. SPIE, 8080, 808022 (2011)

[19] L. Hallo et al., Plasma Phys. Control. Fusion 51, 014001 (2009)

[20] A. Schiavi, S. Atzeni, A. Marocchino, Europhys. Lett. 94, 35002 (2011)

[21] O. Klimo, S. Weber, V. T. Tikhonchuk, J. Limpouch, Plasma Phys. Control. Fusion 52, 055013 (2010)

[22] L. J. Perkins et al. On the Fielding of a High Gain Shock-Ignited Target on the National Ignition Facility in the Near Term (Lawrence Livermore National Laboratory, Livermore, report LLNLTR-428513, 2010) 